\title{
The Drosophila orthologue of the primary ciliary dyskinesia-associated gene, DNAAF3, is required for axonemal dynein assembly
}

\author{
Petra zur Lage ${ }^{1, \pm}$, Zhiyan Xi ${ }^{1, \neq}$, Jennifer Lennon ${ }^{1}$, lain Hunter ${ }^{1, *}$, Wai Kit Chan ${ }^{1}$, Alfonso Bolado Carrancio ${ }^{2}$, \\ Alex von Kriegsheim ${ }^{2}$ and Andrew P. Jarman ${ }^{1, \S}$
}

\begin{abstract}
Ciliary motility is powered by a suite of highly conserved axoneme-specific dynein motor complexes. In humans, the impairment of these motors through mutation results in the disease primary ciliary dyskinesia (PCD). Studies in Drosophila have helped to validate several $P C D$ genes whose products are required for cytoplasmic pre-assembly of axonemal dynein motors. Here we report the characterisation of the Drosophila orthologue of the less-known assembly factor DNAAF3. This gene, CG17669 (Dnaaf3), is expressed exclusively in developing mechanosensory chordotonal (Ch) neurons and the cells that generate spermatozoa, The only two Drosophila cell types bearing cilia/flagella containing dynein motors. Mutation of Dnaaf3 results in larvae that are deaf and adults that are uncoordinated, indicating defective Ch neuron function. The mutant Ch neuron cilia of the antenna specifically lack dynein arms, while $\mathrm{Ca}$ imaging in larvae reveals a complete loss of $\mathrm{Ch}$ neuron response to vibration stimulus, confirming that mechanotransduction relies on ciliary dynein motors. Mutant males are infertile with immotile sperm whose flagella lack dynein arms and show axoneme disruption. Analysis of proteomic changes suggest a reduction in heavy chains of all axonemal dynein forms, consistent with an impairment of dynein pre-assembly.
\end{abstract}

KEY WORDS: Cilium, Flagellum, Drosophila, Ciliopathy, Dynein, Spermiogenesis

\section{INTRODUCTION}

Motile cilia and flagella are highly conserved among eukaryotes ranging from unicellular organisms (e.g. Chlamydomonas, Tetrahymena) to mammals. A motile cilium or flagellum normally comprises a microtubule-based axoneme with nine peripheral microtubule doublets typically surrounding a central pair $(9+2$

\footnotetext{
${ }^{1}$ Centre for Discovery Brain Sciences, Edinburgh Medical School, University of Edinburgh, Edinburgh, UK EH8 9XD, UK. ²Edinburgh Cancer Research

UK Centre, Institute of Genetics and Cancer, University of Edinburgh, Edinburgh, EH4 2XU, UK.

*Present address: Division of Neuroscience and Experimental Psychology, University of Manchester, Stopford Building, Manchester, M13 9PT, UK.

FThese authors contributed equally to this work

§Author for correspondence (andrew.jarman@ed.ac.uk)
}

(D) P.z.L., 0000-0001-7956-1190; Z.X., 0000-0002-5910-5646; I.H., 0000-00024506-9349; W.K.C., 0000-0002-8097-0802; A.B.C., 0000-0003-2330-7489; A.v.K., 0000-0002-4952-8573; A.P.J., 0000-0003-4036-5701

This is an Open Access article distributed under the terms of the Creative Commons Attribution License (https://creativecommons.org/licenses/by/4.0), which permits unrestricted use, distribution and reproduction in any medium provided that the original work is properly attributed.

Received 11 May 2021; Accepted 16 September 2021 structure). These two parts are connected by radial spokes. Some motile cilia lack the central pair and radial spokes ( $9+0$ structure), such as the cilia in the embryonic node. In both $9+2$ and $9+0$ motile cilia, the components responsible for motility are the rows of ciliumspecific (axonemal) dynein motor complexes, visible ultrastructurally as outer and inner dynein arms (ODA and IDA). These complexes drive the sliding of adjacent microtubule doublets to generate cilium movement (Porter, 2017). Dynein motors are large multisubunit complexes comprising heavy chains $(\mathrm{HC},>400 \mathrm{kDa}$ ) for force generation through ATP hydrolysis, intermediate chains (IC, 45$110 \mathrm{kDa}$ ) that scaffold the complex, and light chains (LC, $<30 \mathrm{kDa}$ ) that regulate motor activity (King, 2016).

The autosomal recessive genetic disease, primary ciliary dyskinesia (PCD; MIM 244400), has seemingly complex clinical manifestations including chronic lung infections, progressive damage to the respiratory system, impaired male fertility and abnormal organ symmetry. In recent years the genetic basis of PCD has been intensely studied. The underlying cellular defect of PCD is impaired motility of motile cilia/flagella, but this can result from mutation of one at least 50 different genes (Mitchison and Valente, 2017). Mutations of genes for certain axonemal proteins cause PCD, including several $\mathrm{HC}$ and IC subunits of the beat-generating ODA complex. Of interest are PCD mutations that identify genes required not for dynein components, but for dyneincomplex assembly and transport. Three stages are thought to constitute the biogenesis of dynein arms: cytoplasmic pre-assembly of multiple subunits into dynein complexes (Fok et al., 1994; Fowkes and Mitchell, 1998), transfer of the complexes into the ciliary compartment, and intraflagellar transport (IFT) along the axoneme (Mitchison and Valente, 2017). The pre-assembly of the dynein HCs may be further divided into two phases: the folding and stabilisation of globular head domains of $\mathrm{HCs}$, and the assembly of HCs onto the IC dimer scaffold with incorporation of LCs (Mitchison et al., 2012). A distinct set of around ten proteins, categorised as axonemal dynein assembly factors (DNAAFs), is required for these steps. Their mutations typically cause a combined failure of outer and inner dynein arm localisation on the axonemal microtubules, and the resultant loss of dynein motors can be observed by transmission electron microscopy (TEM) in the cross sections of respiratory epithelial cilia from mutation-bearing PCD patients (Mitchison and Valente, 2017).

Several DNAAF proteins contain domains that are found in HSP90 co-chaperones, such as PIH (Protein Interacting with HSP90), TPR (Tetratricopeptide Repeat), and CS (CHORD-SGT1), leading to the hypothesis that they mediate interactions with chaperones in the cytoplasm for the correct folding/assembly of axonemal dyneins. Several of these assembly factors are hypothesised to form cochaperones similar to the well-known R2TP complex (Kakihara and 
A

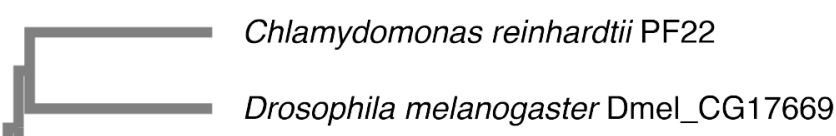

Danio rerio DNAAF3 (predicted)

Xenopus laevis MGC130955

Mus musculus DNAAF3

Homo sapiens DNAAF3
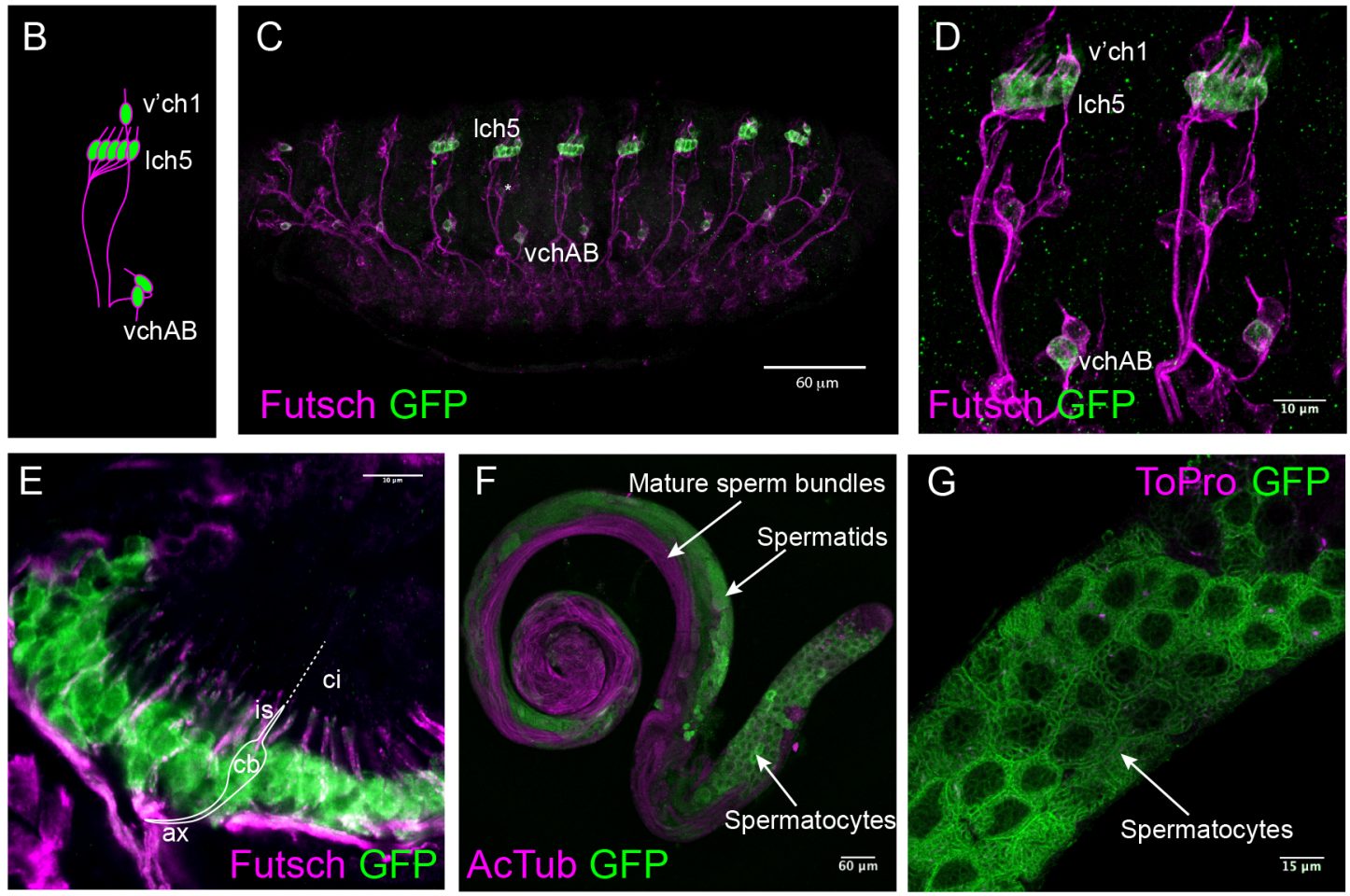

Fig. 1. Expression of CG17669/Dnaaf3. (A) Phylogenetic relationship of CG17669 with other DNAAF3 orthologues, including PF22 from Chlamydomonas. Accession numbers for the proteins are: AEC04845 (for C. reinhardtii PF22), NP_611336 (for Drosophila melanogaster CG17669), XP_003201392 (for Danio rerio predicted DNAAF3), NP_001089839 (for Xenopus laevis MGC130955), NP_001028720 (for Mus musculus DNAAF3), NP_001243643 (for Homo sapiens DNAAF3, isoform 1). (B-G) Expression of Dnaaf3-mVenus fusion gene. (B) Schematic representation of Ch neuron arrangement in an embryonic abdominal segment, with clusters labelled. (C) Stage 16 embryo, showing expression in Ch neurons (anti-GFP, green) relative to sensory neurons (anti-Futsch, magenta). Scale bar: $60 \mu \mathrm{m}$. (D) Higher magnification view of two abdominal segments. Scale bar: $10 \mu \mathrm{m}$. (E) Pupal antenna, showing expression in the Ch neurons of Johnston's organ. Expression is observed in the neuronal cell bodies (cb) and dendrite inner segments (is), but not the cilia (ci, unlabelled by Futsch). Axons (ax) are also indicated. Scale bar: $10 \mu \mathrm{m}$. (F) Adult testis showing fusion gene expression (green) in spermatocytes but not in mature sperm bundles (labelled with anti-acetylated tubulin, magenta). Scale bar: $60 \mu \mathrm{m}$. (G) Higher magnification view of testis showing reticulate pattern of fusion protein in spermatocytes (counter-label for nuclei is To-Pro-3, magenta). Scale bar: $15 \mu \mathrm{m}$.

Houry, 2012; Vaughan, 2014). Such putative 'R2TP-like' complexes may be formed by the PCD proteins DYX1C1 (DNAAF4) and SPAG1 (Knowles et al., 2013; Tarkar et al., 2013) with DNAAF2 (KTU) (Omran et al., 2008) and PIH1D3 (Paff et al., 2017; Tarkar et al., 2013). In Drosophila, WDR92 has been recently linked to R2TP function during dynein assembly (zur Lage et al., 2018). Separately, the PCD protein ZMYND10 is involved in HC stabilisation during which it recruits co-chaperone FKBP8 (Mali et al., 2018). Overall, the molecular evidence supports the notion that many DNAAFs function as cytoplasmic co-chaperones.

However, the interactions and functions of several assembly factors are not clear. DNAAF3 is such a factor. The human protein has no characteristic chaperone-related domains; the functions of its domains (DUF4470 and DUF4471) are not known. Loss-of-function mutations in DNAAF3 were identified initially in three PCD cases that mapped to locus CILD2 (MIM606763), which were characterised by an absence of outer and inner dynein arms in respiratory cilia and consequent ciliary immotility (Mitchison et al., 2012). In Chlamydomonas the DNAAF3 orthologue, PF22, is also required for the presence of ODAs/IDAs in flagella (Mitchison et al., 2012). Based on PF22's cytoplasmic location, and protease sensitivity and antigen exposure of HCs in mutant cells, it was hypothesised that abnormal dynein complexes assemble and accumulate in pf22 mutants, suggesting PF22 functions at a late step in dynein complex 

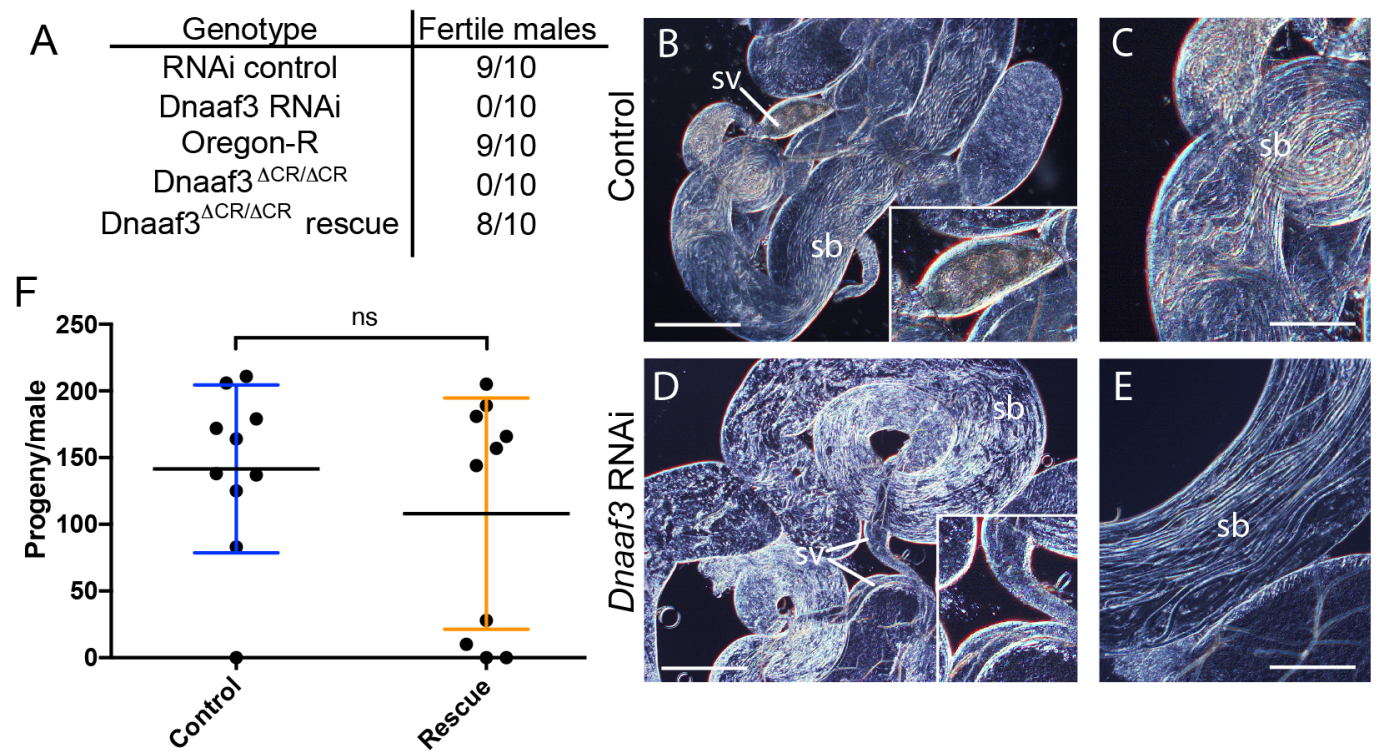

Fig. 2. Dnaaf3 is required for male fertility and sperm motility. (A) Male fertility, presented as number of tested males that produced offspring. (B-E) Adult testes examined by light microscopy. RNAi knockdown of Dnaaf3 results in normal-looking testes with sperm bundles (sb) but no motile sperm are observed in seminal vesicles (sv) (shown at larger size in insets). Scale bar: $50 \mu \mathrm{m}(\mathrm{B}, \mathrm{D})$ or $25 \mu \mathrm{m}(\mathrm{C}, \mathrm{E})$. (F) Fertility of Dnaaf3 ${ }^{\Delta C R}$ homozygotes is substantially rescued by Dnaaf3-mVenus fusion gene. Graph shows number of progeny per male ( $n=10$ males) for control and rescue (Dnaaf3- $m V e n u s /+; D n a a f 3^{\Delta C R} /$ $\left.D_{n a a f 3^{\triangle C R}}\right)$. The average progeny per male is not significantly different by two-tailed Mann-Whitney test $(U=43.00 ; n=10 ; P=0.6299)$.

assembly, possibly in stabilising HCs or in late-stage maturation after cytoplasmic pre-assembly (Mitchison et al., 2012). Apart from further human case reports (Guo et al., 2019), very little further has been published on $D N A A F 3$ or homologues.
Drosophila has recently emerged as a useful metazoan model of ciliary motility. Dynein motors and other ciliary motility components are highly conserved in Drosophila despite the fact that it has only two cell types bearing cilia/flagella containing
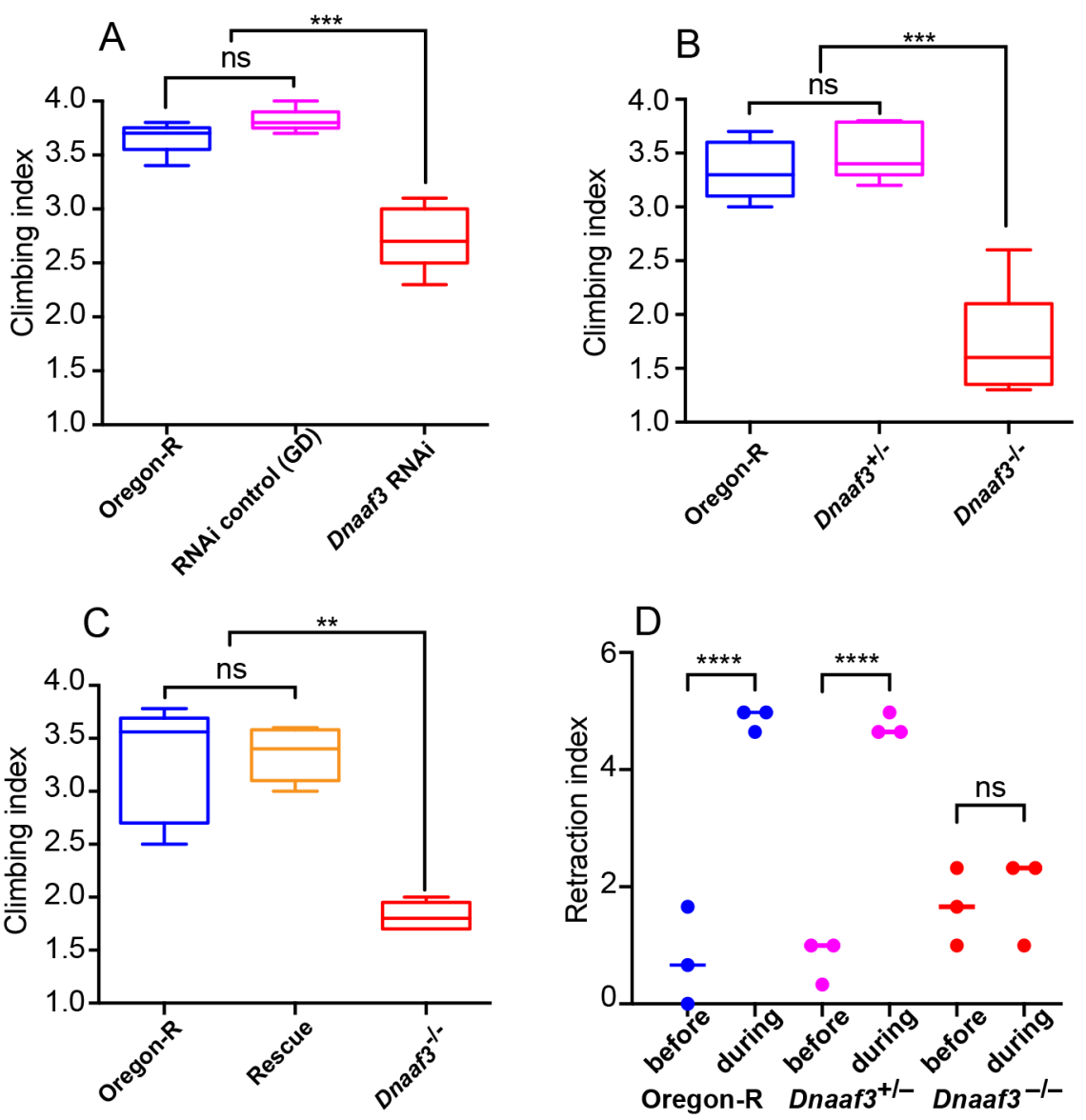

Fig. 3. Dnaaf3 is required for proprioceptive and auditory behaviours. $(\mathrm{A}-\mathrm{C})$ Climbing assays for testing adult incoordination as an indication of proprioceptive function of $\mathrm{Ch}$ neurons. $\mathrm{N}=5$ batches of ten flies for each condition. Data are plotted as median and interquartile range. Significance was determined by Kruskal-Wallis test. (A) Knockdown of Dnaaf3 in sensory neurons results in impaired climbing assay performance. ${ }^{* * *}$ indicates $P=0.0002$. (B) Dnaaf3 $3^{\triangle C R}$ homozygous null mutant flies show impaired climbing assay performance. ${ }^{* * *}$ indicates $P=0.0009$ (C) The performance in the climbing assay of Dnaaf3 $3^{\triangle C R}$ homozygous null mutant flies can be rescued by presence of Dnaaf3-mVenus fusion protein ('rescue'). ${ }^{* *}$ indicates $P=0.0027$ (D) Dnaaf $3^{\perp C R}$ homozygous nul mutant larvae are unresponsive to a $1000 \mathrm{~Hz}$ tone, consistent with defective $C h$ neurons. $n=3$ batches of five larvae in each condition. ${ }^{* * *}$ indicates $P<0.0001$. 
A
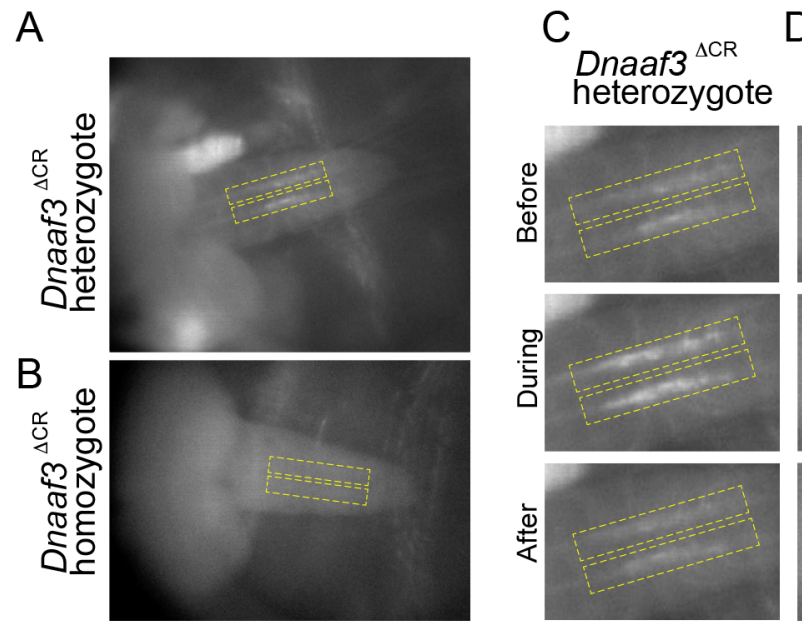

$\mathrm{D}$

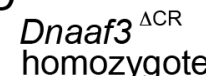

E

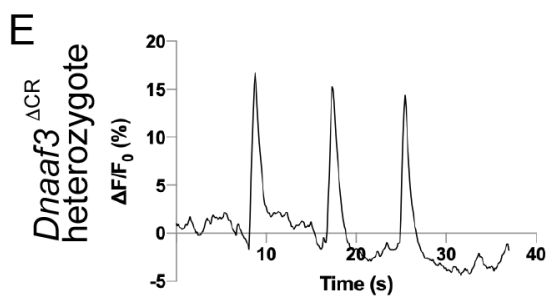

$\mathrm{F}$

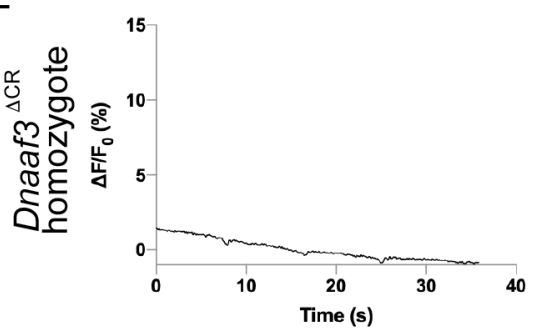

G 15

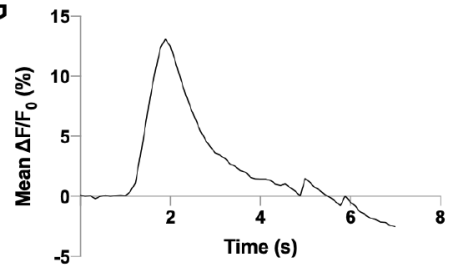

$\mathrm{H}$

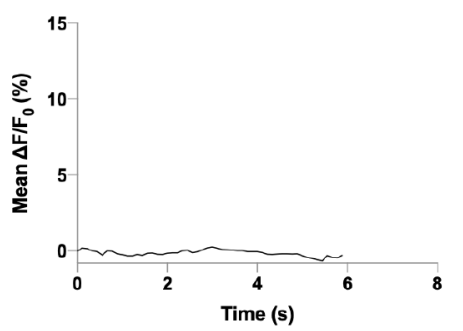

Fig. 4. Response of larval $\mathrm{Ch}$ neurons to vibration stimulation is abolished in Dnaaf3 $3^{\Lambda C R}$ null mutants.

Response to $1024 \mathrm{~Hz}$ vibration stimulation, as fluorescence change in Ch axonal terminals in VNC of semi-intact larval preparations with $\mathrm{Ch}$ neuron-specific Gal4 driving GCaMP expression (iav-Gal4 x UAS-GCaMP6f). (A,B)

Representative frames from video before stimulation for Dnaaf $3^{\triangle C R}$ heterozygote $(A)$ and Dnaaf $3^{\triangle C R}$ homozygote (B). Dashed boxes indicate regions of interest defined to produce traces. (C,D) Representative frames of boxed regions before, during and after stimulation for Dnaaf3 ${ }^{\triangle C R}$ heterozygote (C) and Dnaaf3 ${ }^{\triangle C R}$ homozygote (D). (E,F) Representative traces for Dnaaf3 $3^{\triangle C R}$ heterozygote $(E)$ and homozygote $(F)$ for response $\left(\Delta F / F_{0}, \%\right)$ to three successive $1-\mathrm{s}$ stimuli. $\mathrm{F}_{0}$ is defined as mean $\mathrm{F}$ of: $(0 \mathrm{~s}$ to onset of 1 st peak)+(end of 1 st peak to onset of 2 nd peak) $+($ end of 2 nd peak to onset of 3rd peak). $(\mathrm{G}, \mathrm{H})$ Mean peak response for Dnaaf3 ${ }^{\Delta C R}$ heterozygote (G, mean peak $\Delta \mathrm{F} / \mathrm{F}_{0}=13.54 \% \pm 3.63 ; n=14$ stimulations in five larvae) and Dnaaf3 ${ }^{\Lambda C R}$ homozygote peak $\left(\mathrm{H}\right.$, mean $\Delta \mathrm{F} / \mathrm{F}_{0}=0.25 \%$ $\pm 0.22 ; n=15$ stimulations in five larvae). motility machinery: the sensory cilium of mechanosensory chordotonal $(\mathrm{Ch})$ neurons and sperm flagellum (zur Lage et al., 2019). In both cell types, the motility machinery is critical for function: if defective, mutant flies are deaf and uncoordinated as the motors are required to generate force during $\mathrm{Ch}$ neuron mechanotransduction, and the males are infertile due to immotile sperm (zur Lage et al., 2019). As such, Drosophila is a useful model for identification and analysis of ciliary motility genes, including dynein assembly factors (Diggle et al., 2014; Moore et al., 2013; zur Lage et al., 2018). A single orthologue of DNAAF3 exists in Drosophila: CG17669 (hereafter referred to as Dnaaf3). Here we show that Dnaaf3 is functionally conserved. Tagged Dnaaf3 protein is confined to the cytoplasmic compartment of developing $\mathrm{Ch}$ neurons, and of spermatocytes and spermatids of the testis. RNAi depletion and CRISPR-generated null alleles specifically exhibit phenotypes consistent with ciliary/flagellar immotility with loss of both ODA and IDA. Proteomic analysis of mutants confirmed a specific reduction in abundance of motor proteins, particularly HCs. Unlike reported for Chlamydomonas, it appears that all axonemal dynein types may be affected.

\section{RESULTS}

\section{CG17669 is a DNAAF3 orthologue and is expressed} exclusively in cells bearing motile cilia/flagella

The Drosophila genome has a single homologue of DNAAF3. CG17669 encodes a predicted protein having $27 \%$ amino acid sequence identity and $43 \%$ similarity to human DNAAF3 (Fig. 1A). The CG17669 protein retains the DUF4470 and DUF4471 domains present in human and C. reinhardtii proteins. Therefore, we regard this gene as orthologous and name it Dnaaf3.

To characterise Dnaaf3 expression, we constructed a fly line that expresses a Dnaaf3-mVenus fusion gene, with mVenus in frame with the C-terminus of Dnaaf3 protein (Fig. S1). The construct was designed to include the predicted tissue-specific promoter sequences of Dnaaf 3 , containing predicted binding sites for $\mathrm{Rfx}$ and Fd3F motile cilia transcription factors [consensus binding sequence RYYRYYN(1-3)RRNRAC and RYMAAYA respectively] (Laurençon et al., 2007; Newton et al., 2012). In the embryo, the fusion protein was detected in all differentiating Ch neurons (lch5, v'ch1, and vchAB), but not elsewhere, including other classes of sensory neurons that only have non-motile cilia (Fig. 1B-D). In Ch neurons, Dnaaf3-mVenus protein was localised to cytoplasm (including dendrites) but not the terminal cilia. Adult $\mathrm{Ch}$ neurons develop at the pupal stage during metamorphosis, notably within the developing antenna where they form the proprioceptive/auditory organ called Johnston's organ. In immunofluorescence of pupal antennae, the fusion protein was expressed in the cytoplasm of $\mathrm{Ch}$ neurons exclusively (Fig. 1E).

In testes, the apical tip has spermatogonia that later undergo four mitotic divisions to form primary spermatocytes. The latter go through two meiotic divisions and produce spermatids. Spermatids undergo flagellogenesis within the cytoplasm and the flagellum is 

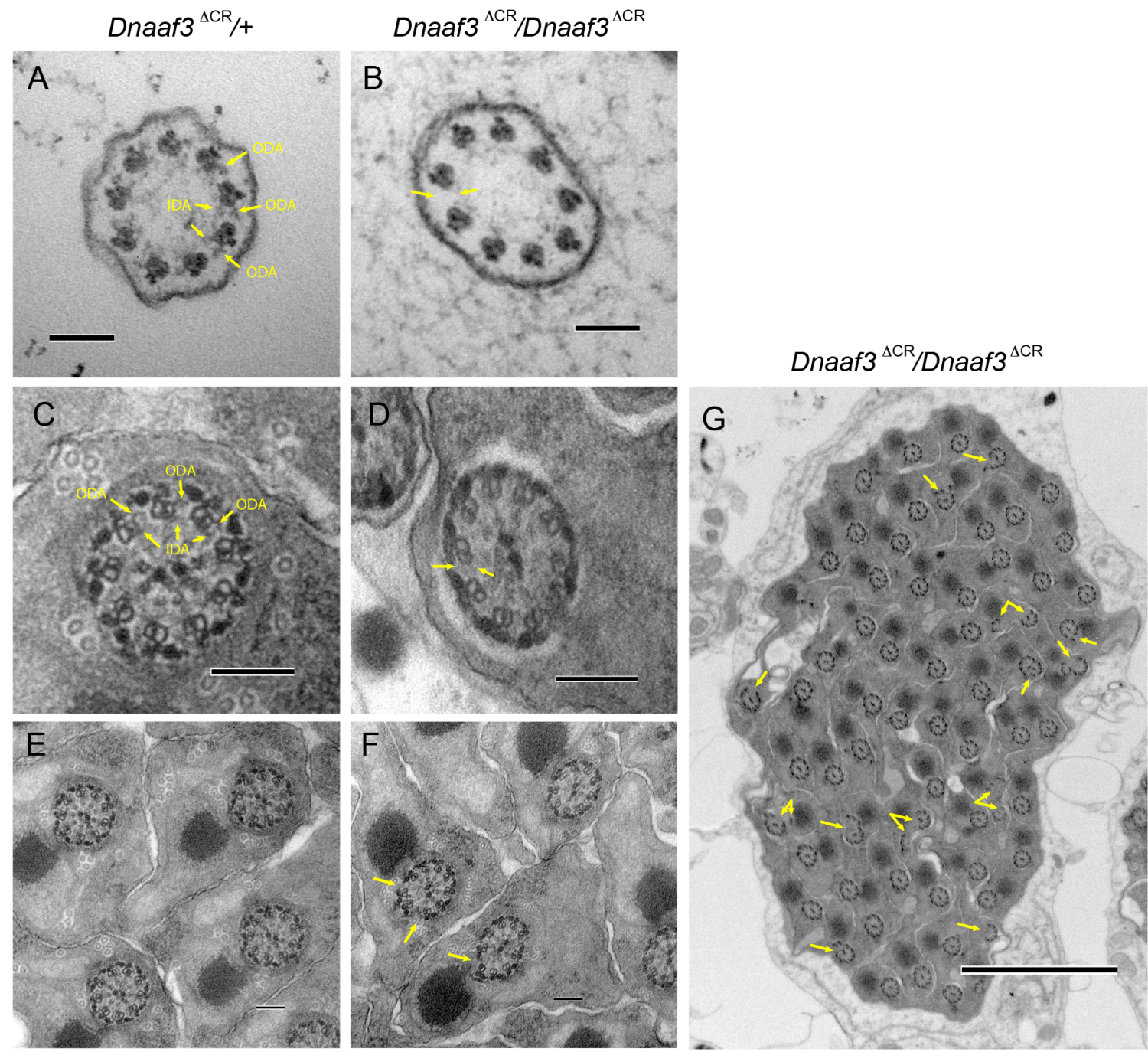
Fig. 5. Loss of dynein arms in Dnaaf3 mutant motile cilia and flagella. Transmission electron micrographs of adult antennal Ch neuron cilia (A,B) and
testis sperm bundles (C-G), transverse sections. (A) Dnaaf3 $3^{\triangle C R}$ heterozygote Ch neuron cilium, showing $9+0$ microtubule doublets with attached outer and inner dynein arms (some arrowed, ODA, IDA). (B) Dnaaf3 $\triangle C R$ homozygote Ch neuron cilium, microtubule doublets are intact, but lack visible ODA and IDA (example of expected location arrowed). (C) Dnaaf $3^{\triangle C R}$ heterozygote sperm flagellum, showing 9+2 structure with ODA and IDA visible on at least some doublets. (D) Dnaaf3 ${ }^{\triangle C R}$ homozygote sperm flagellum, showing regular axonemal structure but ODA/IDA are not visible. (E,F) Lower magnification views, with axonemal splits visible in homozygote (arrowed). (G) Low magnification transverse section of a mature sperm bundle from homozygote, showing generally normal bundle but with frequent axonemal splits (arrows). Scale bars: $100 \mathrm{~nm}(\mathrm{~A}-\mathrm{F}) ; 2 \mu \mathrm{m}(\mathrm{G})$.

then extruded through a process of membrane remodelling and individualisation to form mature sperm that are finally transferred to the seminal vesicle in a motility-dependent manner. The Dnaaf3mVenus fusion protein was detected in locations characteristic of spermatocytes and spermatids, but not in mature sperm bundles (Fig. 1F). It appeared mostly in the cytoplasm in a reticulate pattern (Fig. 1G).

In summary, Dnaaf3 protein is expressed in differentiating motile ciliated cells, and is located to the cytoplasm rather than the cilium/flagellum. The tissue pattern of Dnaaf3 transcription was corroborated by in situ hybridisation: Dnaaf3 mRNA is present specifically in embryonic differentiating $\mathrm{Ch}$ neurons and in spermatocytes of testes (Fig. S2).

\section{Dnaaf3 is required for sperm motility}

To address whether Dnaaf3 is required for flagellar motility, we generated males with testis-specific Dnaaf3 knockdown using the Gal4/UAS system (Bam-VP16-Gal4>UAS-CG17669GD36539
RNAi). In a fertility assay, the knockdown males were observed to mate but produced no offspring (Fig. 2A). The testes of knockdown flies had a normal appearance with apparent sperm bundles (Fig. 2B-E). However, the seminal vesicles were devoid of motile sperm. Even upon crushing of testes, no motile sperm were released from mutant testes (Movies 1, 2).

To confirm this phenotype, we generated a null allele of Dnaaf3 by CRISPR/Cas9 catalysed gene replacement of the ORF with a miniwhite gene. Analysis of homozygous Dnaaf $3^{\triangle C R}$ mutants confirmed that males were viable but sterile, despite being able to mate (Fig. 2A). Their testes were normal in shape and contained normally elongating flagellar bundles of spermatids, but no motile sperms could be observed (Movie 3). Importantly, Dnaaf $3^{\triangle C R}$ infertility could be substantially rescued by the Dnaaf3-mVenus fusion gene. Most rescued males (Dnaaf3-mVenus/+; Dnaaf $3^{\Delta C R} /$ Dnaaf $3^{\Delta C R}$ ) produced progeny, with average fertility not significantly different from that of controls (Fig. 2F). Upon dissection, the seminal vesicles of the rescued males were observed to contain motile sperm (Movie 4). 

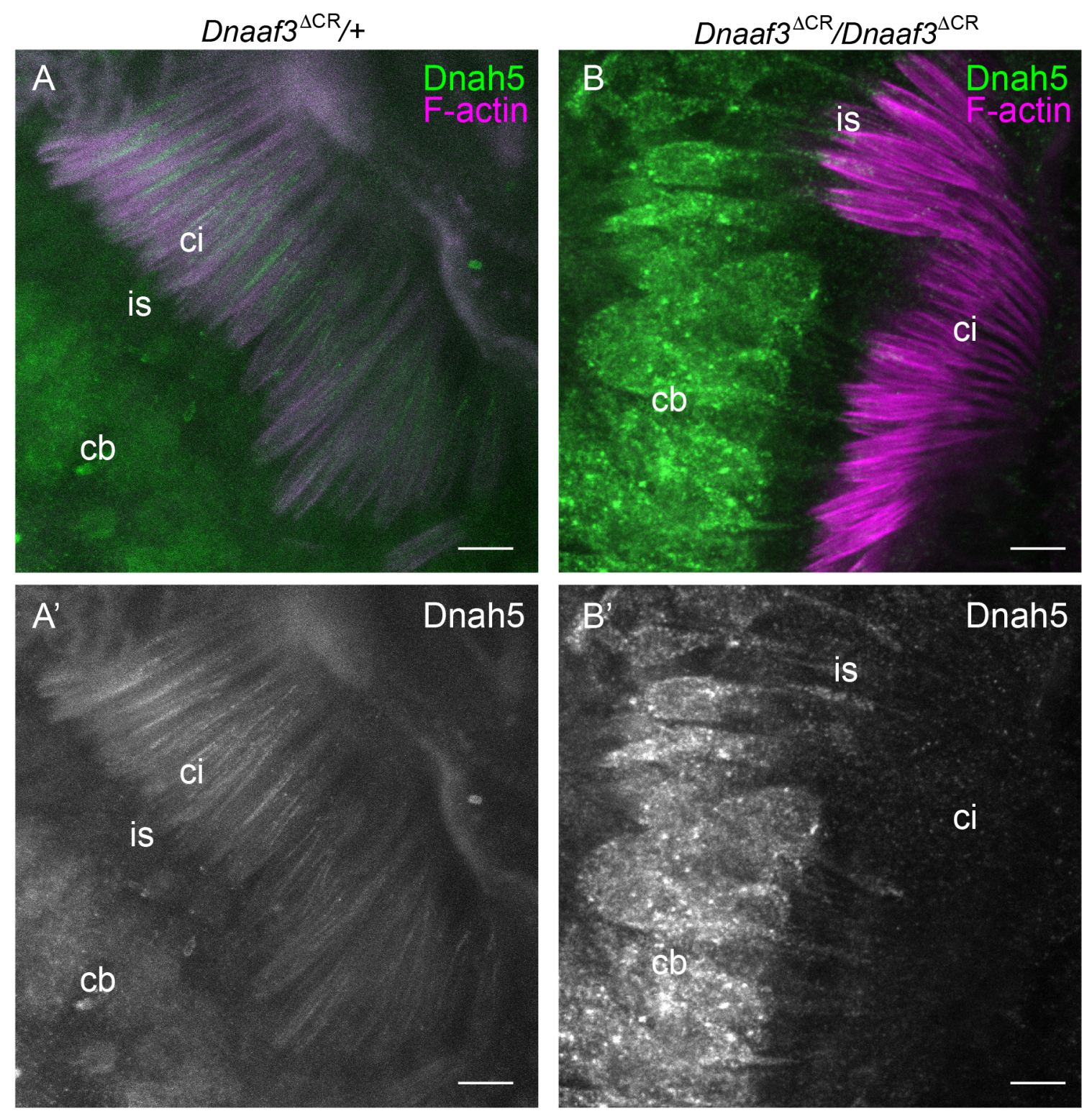

Fig. 6. Loss of dynein heavy chain in Dnaaf3 mutant motile cilia. Immunofluorescence imaging of chordotonal neurons in pupal antenna; Dnah5 protein (green) and phalloidin staining of F-actin (red), which marks the scolopale structures surrounding the ciliary dendrites. (A) Dnaaf3 ${ }^{\Delta C R}$ heterozygote showing Dnah5 protein in cell bodies (cb) and cilia (ci). (B) Dnaaf3 ${ }^{\triangle C R}$ homozygote showing Dnah5 in cell bodies and dendrite inner segments (is) but absent from cilia. $\left(\mathrm{A}^{\prime}, \mathrm{B}^{\prime}\right)$ Corresponding Dnah5 channels only. Number of antennae imaged: heterozygote $n=15$; homozygote $n=10$. Scale bars: $5 \mu \mathrm{m}$.

\section{Dnaaf3 is required for mechanotransduction by Ch neurons}

The $\mathrm{Ch}$ neuron cilium is the site of mechanotransduction by these neurons, and this requires functional axonemal dynein motors (Karak et al., 2015; Newton et al., 2012). To assess whether the proprioceptive function of $\mathrm{Ch}$ neurons requires Dnaaf3, we generated flies with sensory-neuron-specific Dnaaf3 knockdown (scaGal4>UAS-Dcr2/UAS-CG17669 GD36539 RNAi). These knockdown flies were viable but showed uncoordinated locomotion, which was reflected in poor performance in a climbing assay (Fig. 3A). This is consistent with defective $\mathrm{Ch}$ neuron mechanotransduction. Homozygous Dnaaf $3^{\triangle C R}$ mutant flies also showed uncoordinated locomotion in the climbing assay (Fig. 3B). This effect was rescued by the fusion gene (Fig. 3C).

Ch neurons are also the receptors of auditory and vibration stimuli. For the larva, this function is reflected in its response to sound: a brief $1000-\mathrm{Hz}$ tone causes larvae to momentarily retract, a response that depends on $\mathrm{Ch}$ neuron function (zur Lage et al., 2018) (Fig. 3D). In this assay, we observed that homozygous Dnaaf $^{\triangle C R}$ larvae do not respond to a $1000-\mathrm{Hz}$ tone (Fig. 3D), suggesting failure in auditory/vibration mechanotransduction in $\mathrm{Ch}$ neurons.

To determine directly whether the larval Ch neurons can respond to a tone stimulus, we visualised $\mathrm{Ch}$ neuron activation in larval fillet preparations by recording calcium changes in their axon termini using a genetically supplied GCaMP calcium reporter (iavGal4, UAS-GCaMP6f) (Fushiki et al., 2013). Freshly dissected 3rd instar larval pelts were stimulated by $1024-\mathrm{Hz}$ vibrations supplied by a tuning fork, and the GCaMP response recorded by imaging. In Dnaaf $3^{\triangle C R}$ heterozygote larvae, Ch neurons showed robust, short duration peaks in $\mathrm{Ca}$-induced fluorescence upon vibration stimulus (mean peak response to stimulation, $\Delta \mathrm{F} / \mathrm{F}_{0}: 13.54 \% \pm 3.63 ; n=14$ 
Table 1. Ciliary motility proteins whose abundance changes in Dnaaf3 mutant testes $(P<0.05)$, sorted by $P$ value

\begin{tabular}{|c|c|c|c|c|c|}
\hline Majority protein IDs & Gene names & LFQ control & LFQ Dnaaf3 & -log2 ratio & $-\log 10 P$ value \\
\hline Q9VWZ3 & Dhc16F & 1.01829888 & 0.041609539 & 4.613103 & 3.265697 \\
\hline A8Y5B7 & $\mathrm{kl}-3$ & 6.80093659 & 0.430428367 & 3.981888 & 3.182219 \\
\hline A1ZB91 & Dnaaf3 & 1.2915202 & 0.081774953 & 3.981267 & 3.103016 \\
\hline Q5LJP0 & $\mathrm{kl}-2$ & 0.78943234 & 0.224258622 & 1.815652 & 2.822993 \\
\hline Q9VU57 & Cfap43 & 0.55106365 & 0.068451057 & 3.009074 & 2.674799 \\
\hline Q9VJC6 & Dhc36C & 2.82836711 & 0.163285342 & 4.114502 & 2.494444 \\
\hline Q7KVA7 & Dhc62B & 1.00184398 & 0.157084244 & 2.673047 & 2.321502 \\
\hline Q9VZ77 & Dnah3 & 1.08739055 & 0.119223228 & 3.189133 & 2.309416 \\
\hline Q9VKJ5 & Dyx1c1 & 0.05987797 & 0.20546445 & -1.77879 & 2.303495 \\
\hline Q0E8T7 & CG34124 & 1.18667573 & 0.490089781 & 1.275808 & 2.25354 \\
\hline Q5LJN5 & $\mathrm{kl}-5$ & 8.19193092 & 2.814315855 & 1.541419 & 2.208549 \\
\hline Q9VAV5 & Dhc98D & 1.83012501 & 0.712779034 & 1.360415 & 2.116203 \\
\hline Q9VM21 & gudu & 1.62031671 & 0.232234203 & 2.802623 & 2.02667 \\
\hline Q9VVM7 & Wdr92 & 0.27889713 & 0.453183439 & -0.70036 & 1.994956 \\
\hline Q9VJY4 & Dnai2 & 0.67582694 & 0.303152945 & 1.156608 & 1.965192 \\
\hline Q9W0U9 & Dic61B & 0.22810593 & 0.14967333 & 0.607887 & 1.814405 \\
\hline Q9VR52 & Lrrc6 & 0.02411973 & 0.09266751 & -1.94185 & 1.793124 \\
\hline Q9W0F0 & robl62A & 0.25048477 & 0.039268251 & 2.673288 & 1.774009 \\
\hline Q9VCN4 & Ccdc114 & 1.08124333 & 0.208368775 & 2.37548 & 1.746445 \\
\hline Q9VGG6 & Dnali1 & 3.88414271 & 1.440937117 & 1.430589 & 1.638454 \\
\hline Q4V516 & lobo & 0.4429829 & 0.227454095 & 0.961676 & 1.597102 \\
\hline Q9W1V2 & CG3085 & 4.91455102 & 1.939847136 & 1.341117 & 1.576174 \\
\hline Q9VIY1 & CG10750 & 0.03495738 & 0.007402311 & 2.23955 & 1.554517 \\
\hline Q8T3Z0 & Tektin-C & 2.44201942 & 1.224471976 & 0.995915 & 1.457508 \\
\hline Q9W168 & CG16837 & 0.06873574 & 0.028035895 & 1.293785 & 1.453004 \\
\hline Q9W2A3 & CG13501 & 0.13118274 & 0.07513098 & 0.804098 & 1.408852 \\
\hline
\end{tabular}

stimuli in five larvae) (Fig. 4A,C,E). In contrast, Ch neurons from homozygous Dnaaf $3^{\Delta C R}$ larvae did not show a robust response (mean peak $\Delta \mathrm{F} / \mathrm{F}_{0}: 0.25 \% \pm 0.22 ; n=15$ stimuli in five larvae) (Fig. 4B,D,F). There was a significant difference between mean peak $\Delta \mathrm{F} / \mathrm{F}_{0}$ in heterozygotes and homozygotes (unpaired $t$-test, $P \leq 0.0001)$.

Overall, null mutant individuals have multiple phenotypes consistent with impaired motility of cilia/flagella. This strongly supports the hypothesis that Drosophila Dnaaf3 is required for dynein motor assembly or function.

\section{Ultrastructural analysis shows specific absence of dynein arms from cilia and flagella}

Human $D N A A F 3$ mutations are characterised by ultrastructural absence of ciliary ODA/IDA, consistent with a failure in cytoplasmic pre-assembly of dynein motor complexes. We used TEM to analyse Ch neuron cilia and sperm flagella in Dnaaf $3^{\Delta C R}$ mutant adult flies. In the antennal Johnston's organ, transverse sections of $\mathrm{Ch}$ neuron cilia revealed a normal axonemal structure of 9+0 microtubule doublets, but ODA and IDA both appeared lacking (Fig. 5A,B). The loss of ODA was further examined in pupal Johnston's organ Ch neurons by immunofluorescence using an antibody generated against the Drosophila orthologue of the ODA HC, Dnah5 (CG9492). This heavy chain is expressed exclusively in Ch neurons (zur Lage et al., 2018) and localises to the ciliary proximal zone (Fig. 6A). In the cilia of Ch neurons in Dnaaf $3^{\Delta C R}$ homozygote antennae, Dnah5 protein was observed to be strongly reduced or absent (Fig. 6B).

In the testes of Dnaaf $3^{\triangle C R}$ homozygote males, TEM showed that sperm flagellum axonemes were largely normal, but strongly lacked ODA/IDA (Fig. 5C-F). In the sperm bundles, axonemal breakage was also observed, whereby one or more microtubule doublets break away from the axoneme (Fig. 5F,G). Such a phenotype has been observed for most other homologues of dynein assembly factors (zur Lage et al., 2018).

\section{Proteomic analysis of mutant testes reveals specific reduction in abundance of dynein heavy chains}

In many dynein assembly factor mutants, failure in pre-assembly and localisation of dynein motors appears to result in instability of some dynein subunits, particularly HCs (Mitchison et al., 2012). In Drosophila, testis protein abundances have previously been assayed by mass spectrometry in order to characterise the phenotype of the dynein assembly factor, $W d r 92$ (zur Lage et al., 2018). We similarly carried out mass spectrometry on adult testes from Dnaaf $3^{\Delta C R}$ mutant and control flies. By label-free quantitative MS, we detected 5549 proteins. To examine ciliary proteins, we focused on the MS data for homologues of proteins associated specifically with motile cilia (dynein motors, nexin-dynein regulatory complex, radial spokes, etc; zur Lage et al., 2019). Of these 92 candidate proteins, 81 were detected as being present in the MS data but we excluded 17 that were not reliably detected (Table S1). Interestingly, in wild-type testes, dynein assembly factors and chaperones are among the most abundant proteins, suggesting that dynein assembly is a major activity in spermatozoan differentiation (Table S1).

Among the proteins detected is Dnaaf3 itself, and as expected this protein shows a large depletion in the Dnaaf $3^{\Delta C R}$ mutant testes (Table 1). Of the ciliary motility proteins detected, 26 showed significant difference in abundance in Dnaaf $3^{\Delta C R}$ mutant testes (>1.5-fold) (Table 1, Fig. 7A). Most of these were depleted, and notable among these are axonemal dynein $\mathrm{HCs}$, of which all eight chains are significantly depleted (Table 2, Fig. 7B,C). A few ciliary proteins were enriched, and these are notably a subset of other dynein assembly factors (Wdr92, Dyx1c1, Lrrc6).

\section{DISCUSSION \\ Dnaaf3 has conserved functions in ciliary axonemal dynein assembly}

Functional analysis indicates that Drosophila Dnaaf3 (CG17669), like human $D N A A F 3$, is required for axonemal dynein complex assembly and localisation. Moreover, it is required for dynein 
A

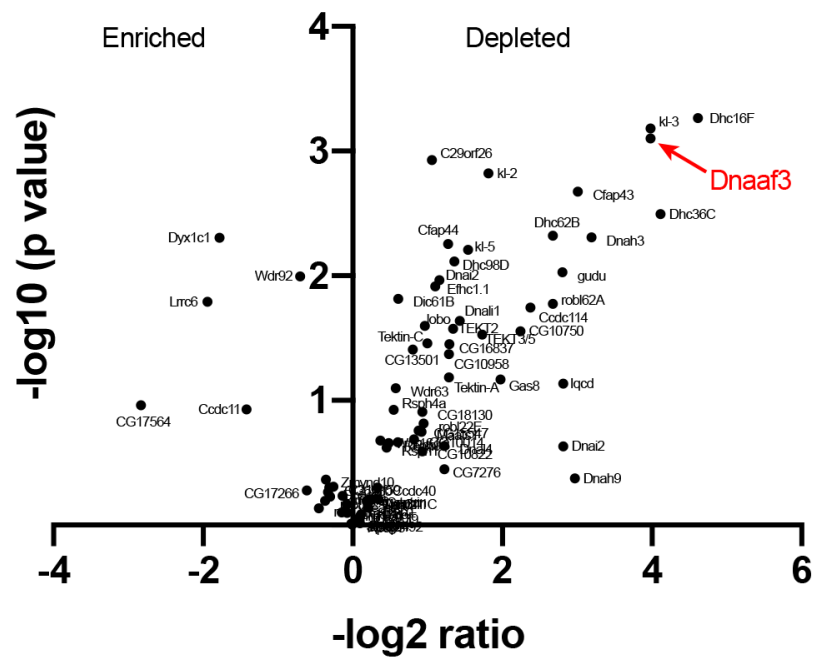

B

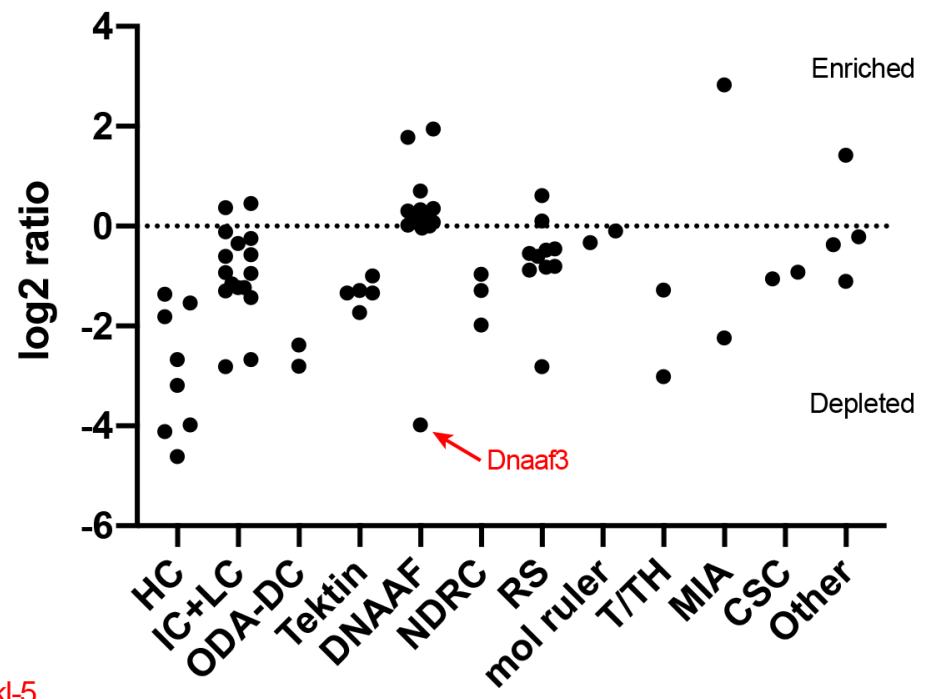

C
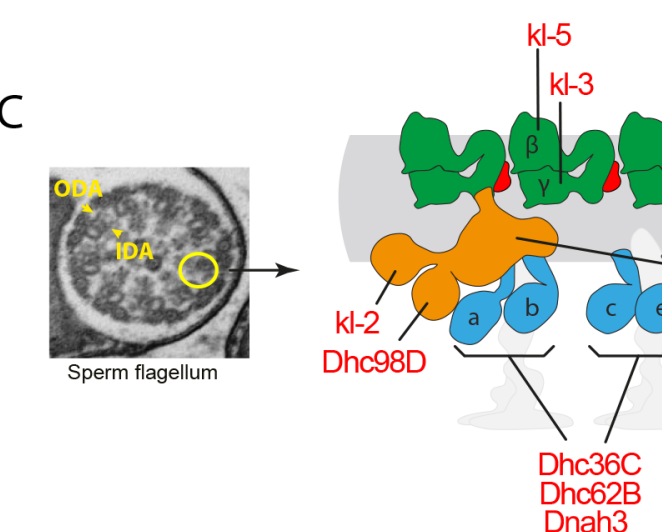

Outer dynein arms (ODA)

Fig. 7. Dynein motor subunits are depleted in Dnaaf3 mutant testes. (A) Volcano plot of motile cilia-associated proteins detected by MS in testes. To the left of the $\mathrm{Y}$ axis are proteins that are more abundant in homozygote testes (enriched); to the right are proteins that are less abundant (depleted). Dnaaf3 protein itself is strongly depleted as expected (red). (B) Summary of protein abundances for ciliary motility proteins. Below the dotted 0 line are proteins depleted in the mutant. HC, dynein heavy chain; IC, dynein intermediate chain; LC, dynein light chain; ODA-DC, outer dynein arm docking complex; DNAAF, dynein assembly factor; NDRC, nexin-dynein regulatory complex; RS, radial spoke; mol ruler, molecular ruler complex (CCDC39/40); T/TH, tether/tetherhead complex; MIA, modulator of inner arm; CSC, calmodulin-spoke complex. (C) Schematic of the arrangement of dynein motor complexes along one 96$\mathrm{nm}$ repeating unit of an axonemal microtubule in the sperm flagellum (adapted from Lin et al., 2014; zur Lage et al., 2019). Colours indicate ODA (green), ODA-DC (red), double-headed IDA $f$ (orange), single headed IDAs a-g (blue). The basal part of IDA $f$ is also called the outer-inner dynein linker (OID). The heavy chains of all subtypes (red protein names) are depleted in the mutant testes.

localisation in both IFT-dependent motile cilia (Ch neuron dendrites) and IFT-independent flagella (sperm). The Dnaaf3 phenotype, including the lack of visible ODA and IDA on TEM images, suggests a complete loss of dynein arms from mature cilia/ flagella. Together with the cytoplasmic location of Dnaaf3 protein, these features are consistent with a role in the cytoplasmic preassembly of dynein complexes, as has been proposed for the human and Chlamydomonas orthologues.

In PCD patients with $D N A A F 3$ mutations, respiratory cilia did not localise several subunits associated with ODA (DNAH5, DNAH9, and DNAI2) or a subunit associated with a subset of single-headed IDAs (DNALI1). Chlamydomonas pf 22 mutant flagella similarly showed loss of subunits associated with ODA and the single-headed IDA forms $b$ and $c$ (see Fig. 7C for summary of dynein forms). However, a subunit of the double-headed form, IDA f (IC140), was still present in mutant flagella (Mitchison et al., 2012) suggesting that this IDA form did not require $D N A A F 3$ for its assembly. However, in our proteomic analysis we found reduction in abundance of all sperm-expressed HCs in the Dnaaf3 mutant testes. Notably, this includes the two HCs of double-headed IDA f, both of which were prominently reduced. To reconcile this, we suggest that the presence of IC140 in Chlamydomonas pf 22 mutant flagella does not represent a complete IDA f complex, but just the IC-LC part. Indeed, this IC-LC part is thought to function independently as a motility regulator and has been called the IC138 subcomplex (Bower et al., 2009) or outer-inner dynein linker (OID) (Oda et al., 2013). We suggest, therefore, that the IC138 subcomplex/OID can assemble and localise in the absence of HCs, and moreover its assembly does not require DNAAF3. Interestingly, a parallel to this can be seen in wild-type Drosophila Ch neurons: we previously showed that these cells express IC138 subcomplex/ OID subunits but not the corresponding IDA f HCs, supporting the presence of an independent IC138 subcomplex/OID in these cilia (zur Lage et al., 2019).

Based on protease sensitivity and antigen presentation in pf22 mutants, it was hypothesised that abnormal dynein complexes accumulate, leading to the suggestion that PF22 functions at a late step in assembly, possibly in stabilising HCs or in late-stage 
Table 2. Dynein heavy chains in Dnaaf $3^{\Delta C R}$ mutant testis proteome

\begin{tabular}{|c|c|c|c|c|c|}
\hline $\begin{array}{l}\text { Protein } \\
\text { accession }\end{array}$ & Gene & $\begin{array}{l}\text { Human } \\
\text { orthologue }\end{array}$ & $\begin{array}{l}\text { Dynein } \\
\text { complex }\end{array}$ & $\begin{array}{l}\text {-log2 } \\
\text { (ratio) }\end{array}$ & $\begin{array}{l}\text {-log10 } \\
\text { ( } P \text { value) }\end{array}$ \\
\hline \multicolumn{6}{|l|}{ ODA } \\
\hline A8Y5B7 & $k l-3$ & DNAH8 & ODA $\beta$ & 3.981888 & 3.182219 \\
\hline Q5LJN5 & $k l-5$ & DNAH17 & ODA $\gamma$ & 1.541419 & 2.208549 \\
\hline \multicolumn{6}{|c|}{ IDA (single headed) } \\
\hline Q9VJC6 & Dhc36C & DNAH7 & IDA a,b,c & 4.114502 & 2.494444 \\
\hline Q9VZ77 & Dnah3 & DNAH3 & IDA a,b,c & 3.189133 & 2.309416 \\
\hline Q7KVA7 & Dhc62B & DNAH12 & IDA $a, b, c$ & 2.673047 & 2.321502 \\
\hline Q9VWZ3 & Dhc16F & DNAH6 & IDA d,e & 4.613103 & 3.265697 \\
\hline \multicolumn{6}{|c|}{ IDA (double headed) } \\
\hline Q9VAV5 & Dhc98D & DNAH10 & IDA $f$ & 1.360415 & 2.116203 \\
\hline Q5LJP0 & $k l-2$ & DNAH2 & IDA f & 1.815652 & 2.822993 \\
\hline
\end{tabular}

maturation after cytoplasmic pre-assembly (Mitchison et al., 2012). Moreover, cytoplasmic abundances of HCs were not affected, whereas IC2 was increased. This contrasts with other dynein assembly factors, which often show reduction in cytoplasmic levels consistent with protein instability. This was also taken to support the idea that DNAAF3 acts at a different step from other dynein assembly factors. In contrast, in Drosophila mutant testes we found reduction in total amounts of many motor components but particularly HCs. We cannot, however, be certain that this reflects cytoplasmic levels during dynein pre-assembly.

Several DNAAFs have been found to interact during their function, such as DYX1C1/PIH1D3 and ZMYND10/LRRC6 (Moore et al., 2013). Several DNAAFs (including DYX1C1, PIH1D3, SPAG1, DNAAF2) are thought to form complexes with Reptin and Pontin, similar to the co-chaperone R2TP. In contrast, investigations of protein interactions of DNAAF3 homologues have not so far proved informative (Mitchison et al., 2012). Similarly, we have not been able to identify proteins that interact with Drosophila Dnaaf3 by AP-MS (unpublished observations). In the samples, the MS/MS count of Dnaaf3 was large, but few associated proteins were detected, and none appeared interesting from the point of view of dynein biology, protein folding, or transport. The inference is that DNAAF3 protein interactions are too transient for detection by immunoprecipitation methods. The molecular function of DNAAF3 homologues therefore remains mysterious, perhaps suggesting a role not closely related to other DNAAFs. It is intriguing, therefore, that Dnaaf3 fusion protein appeared in a reticulate pattern in spermatocytes, a pattern that has not been observed for other Drosophila DNAAFs so far (Diggle et al., 2014; Moore et al., 2013; zur Lage et al., 2018). It remains to be seen whether this reflects a difference in molecular function.

\section{Axonemal dyneins are required for mechanosensation by Ch neurons}

By $\mathrm{Ca}$ imaging, we show that the Ch neurons of Dnaaf3 mutant larvae are unable to respond to a vibration stimulus, and the larvae correspondingly do not respond behaviourally to a tone stimulus. This confirms that axonemal dynein motors in Ch neuron cilia are absolutely required for the mechanotransduction process in hearing, supporting previous findings in adult auditory $\mathrm{Ch}$ neurons, in which mutation of ODA IC or IDA HC subunits caused defects in auditory mechanotransduction (Karak et al., 2015). The molecular role of the axonemal dyneins in hearing is not fully determined, but they are likely responsible for active mechanical amplification of stimuli as well as adaptation within $\mathrm{Ch}$ neuron cilia (Albert and Göpfert, 2015).
Dnaaf3 mutant flies also show a partial reduction in proprioceptive ability, but this does not appear to be as severe as observed for $f d 3 F$ mutant flies, in which Ch neurons are exclusively and completely non-functional (Newton et al., 2012). Thus, for the detection of stretch, some $\mathrm{Ch}$ neuron function may remain in the absence of ciliary motors. Compared with proprioception, the loss of hearing may relate to the greater need to amplify the tiny $\mathrm{Ch}$ ciliary displacements that result from vibration stimuli, as well as the role of mechanical positive feedback in frequency selectivity.

\section{A role for axonemal dynein motors in axoneme stabilisation during IFT-independent flagellogenesis?}

Like most cilia, ciliogenesis in Drosophila sensory neurons occurs from a basal body docked at the plasma membrane, with the axoneme extending within a bounding membrane. This requires IFT for transport of components within the extending ciliary compartment (i.e., ciliogenesis is compartmentalised). In contrast, Drosophila sperm flagellogenesis occurs by axonemal extension within the cytosol in a process that does not require IFT (Basiri et al., 2014; Fingerhut and Yamashita, 2020; Han et al., 2003; Sarpal et al., 2003; Vieillard et al., 2016). Microtubule doublet extension occurs within a membrane 'ciliary cap' but the growing axoneme is pushed out into the cytoplasm (Basiri et al., 2014). Once outside the ciliary cap, motors are docked from the cytoplasm (Fingerhut and Yamashita, 2020). Subsequently, sperm undergo a process of individualisation which strips off cytoplasm and surrounds the flagellum axoneme with membrane (Basiri et al., 2014; Fabian and Brill, 2012).

Our analysis suggests that Drosophila Dnaaf3 is specifically required for axonemal dynein assembly. However, in addition to dynein arm loss, Dnaaf3 sperm flagella show axoneme disruption with doublets frequently splitting off. Such a phenotype has been noted in all Drosophila dynein assembly factor mutants analysed so far (tilB, Zmynd10, Heatr2, Spag1, Wdr92) (Diggle et al., 2014; Kavlie et al., 2010; Moore et al., 2013; zur Lage et al., 2018). Moreover, axonemal breakage is seen in mutants of many axonemal dynein subunits themselves (including $g u d u, k l-2, k l-3, k l-5$, Dhc98D) (Steinhauer et al., 2019). We suggest that in the absence of a bounding membrane during flagellogenesis, dynein complexes provide a temporary doublet 'cross-linking' function to maintain axoneme stability prior to being surrounded by membrane during sperm individualisation. When dynein attachment is compromised, the microtubule doublets will be vulnerable to separation prior to individualisation. This then has a knock-on effect of disrupting individualisation, which also appears compromised in mutants with disrupted axoneme (Fatima, 2011; Steinhauer et al., 2019).

If this view that axonemal dyneins are required to stabilise the immature axoneme is correct, then it may also help to explain the discovery that several axonemal dynein $\mathrm{HCs}$ are translated in phase condensates close to the site of axoneme extension (Fingerhut and Yamashita, 2020), which may facilitate synthesis and delivery of dyneins to the unstable immature axoneme. Such 'kl-granules' contain Reptin and Pontin in addition to HC mRNAs, which has led to the suggestion that they are the site dynein complex pre-assembly concomitant with translation (Fingerhut and Yamashita, 2020). While such an arrangement would clearly be beneficial, it remains to be seen whether R2TP-like DNAAFs colocalise with Reptin and Pontin in kl-granules.

Since a similar cytosolic mode of assembly is also proposed for the formation of mammalian sperm flagella (Avidor-Reiss and Leroux, 2015), it will be interesting to determine whether defective dynein assembly results in secondary defects in axonemal stability and sperm individualisation of mammalian sperm. 


\section{MATERIALS AND METHODS}

Fly stocks

All fly strains were maintained on standard media at $25^{\circ} \mathrm{C}$. The following stock were obtained from the Bloomington Stock Center: $y^{I}$ $w^{*} \mathrm{P}\left\{\mathrm{y}^{\mathrm{t} 7.7}=\right.$ nos-phiC31 int.NLS $\} \mathrm{X} ; \mathrm{P}\left\{\mathrm{y}^{\mathrm{t7} .7}=\right.$ CaryP $\}$ attP40 (\#79604), $y^{l}$ $\mathrm{M}\{\mathrm{RFP} 3 \mathrm{xP} 3 . \mathrm{PB}] \mathrm{GFP} \mathrm{E}^{\mathrm{E} .3 \times \mathrm{P} 3}=$ vas-Cas9 $\} \mathrm{ZH}-2 \mathrm{~A} \quad(\# 52669), \quad w^{1118} / \mathrm{FM} 7 \mathrm{c}$ (\#51323), $w^{1118}$ (\#3605), $w^{*} \mathrm{P}\left\{\mathrm{w}^{+\mathrm{mC}}=\mathrm{iav}-\mathrm{Gal} 4 . \mathrm{K}\right\} 3 \quad$ (\#52273), $w^{*}$ $\mathrm{P}\left\{\mathrm{y}^{+\mathrm{t} 7.7} \mathrm{w}^{+\mathrm{mC}}=5 \mathrm{xUAS}-\mathrm{GCaMP} 6 \mathrm{f}\right\} \mathrm{attP} 2 \quad(\# 91989)$, and $\mathrm{P}\left\{\mathrm{w}^{+\mathrm{mC}}=\mathrm{UAS}-\right.$ Dcr-2.D 1 (\#24648). The w; Tft/CyO; Bam-VP16-Gal4 was a gift from $\mathrm{H}$. White-Cooper and the sca-Gal4 line from M. Mlodzik. The lines for the RNAi experiments, GD control (ID 60000) and CG17669 RNAi (ID GD36538) were obtained from the Vienna Drosophila Resource Center (VDRC) (Dietzl et al., 2007).

\section{In situ hybridisation}

In situ hybridisation on overnight wild-type embryos was carried out according to (zur Lage et al., 2019) and on 3-4-day old adult testes according to (Morris et al., 2009). The mounted slides were photographed on an Olympus AX70 upright microscope. The RNA probe for Dnaaf3 was generated by T7 RNA polymerase in vitro transcription from a gene fragment isolated by PCR using the primers CAGACTGGACCGTTTTGTGG and GTAATACGACTCACTATAGGGCCCACATGTTCTTGCCGTTGA. The latter includes the T7 RNA polymerase recognition sequence.

\section{mVenus fusion gene construction}

The CG17669::mVenus fusion construct was designed to include the CG17669 ORF and upstream regulatory sequences, with mVenus fused inframe C-terminally. PCR primers used to amplify the CG17669 sequence were GGGGACAAGTTTGTACAAAAAAGCAGGCTAGTGTTGCATACGAAAGGGT and GGGGACCACTTTGTACAAGAAAGCTGGGTCTGGGAAGTTAGTTTTTGGTT. The cloning was performed according to the Gateway two-step recombination system (Life Technologies). After the $\mathrm{BP}$ reaction, the construct was transferred into the pBID-UASC-GV vector (Wang et al., 2012) via the LR reaction in order to generate the expression clone pBID-UASC-CG17669::mVenus. The construct was subsequently microinjected into the attp40 line.

\section{CRISPR/Cas9-guide RNA expression construction}

A CG17669 CRISPR/Cas9 mutant was constructed by a mini-white gene substitution as described by (zur Lage et al., 2018). Two pairs of sense and antisense oligonucleotides (oligos) were designed for guide RNAs (gRNA I and II, respectively) using the CRISPR optimal target finder (http://tools. flycrispr.molbio.wisc.edu/targetFinder/index.php) based on the CG17669 sequence. Guides were, gRNA I: CTTCGGAGGATGAGCATGCACGCG and AAACCGCGTGCATGCTCATCCTCC; gRNA II: CTTCGAACCTGCTGAAGAGTATAC and AAACGTATACTCTTCAGCAGGTTC. The guides were phosphorylated and annealed before cloning into the $B b s \mathrm{I}$ restriction site of pBFv-U6.2 and pBFv-U6.2B (Kondo and Ueda, 2013), respectively. For both guides to be expressed from the same plasmid, an EcoRI-NotI fragment from pBFv-U6.2 containing the gRNA I sequence was subsequently cloned into the gRNA II-containing plasmid pUBFv-U6.2B. For homology-directed repair, homology arms were amplified from genomic DNA using the following primer pairs: left arm - GATGCATGCCGTCAAACAACAGCCAAAAG and GATGGTACCACTTCCACTTCCACCCTGGT; right arm - GGGAGATCTTACTGGTCTAGTAATTGAAG and CAACTCGAGATCCATAATTGCTGGCAACC. The left homology arm was cloned into the $S p h$ I and Asp718I restriction sites (underlined) of the $5^{\prime}$ multiple cloning site of pRK2 (Huang et al., 2008), whereas the right homology arm was cloned into the BglII and XhoI sites of the $3^{\prime}$ multiple cloning site of the same vector. The guide RNA and the homology arm constructs were injected into the vas-Cas9 line. Substitution of the $C G 17669$ ORF by mini-white (the Dnaaf $3^{\Delta C R}$ allele) was confirmed by sequencing.

\section{Immunofluorescence}

Embryo and pupal antenna immunohistochemistry was carried out according to (zur Lage et al., 2018). The fixing and staining of
Drosophila testis was described in Sitaram et al., 2014 (Sitaram et al., 2014). The following primary antibodies were used: rabbit anti-GFP antibody (1:500, Life Technologies, A11122), mouse anti-Futsch antibody (1:200, Developmental Studies Hybridoma Bank, 22C10-s), mouse antiacetylated tubulin (1:1000, Sigma, T6793) and rabbit anti-Dnah5 antibody (1:2000, see below) and the secondary antibodies were goat anti-Rabbit antibody (1:500, Alexa Fluor 488, Life Technologies, A11008) and goat anti-Mouse antibody (1:500, Alexa Fluor 568, Life Technologies, A11019). DNA in adult testes was stained with To-Pro-3 (1:1000, Life Technologies, T3605) solution in the dark for $15 \mathrm{~min}$. After several washes, the samples were mounted on slides with $85 \%$ glycerol and $2.5 \%$ propyl gallate (SigmaAldrich, P3130). The slides were imaged using a Zeiss LSM-5 PASCAL/ Axioskop 2 confocal microscope and processed with Fiji.

\section{Dnah5 (CG9492) antibody generation}

A 744-bp sequence was amplified from exon 5 of the Drosophila CG9492 gene and cloned into the EcoRI restriction site (underlined) of the expression vector pRSET-A using the following primers: left GGGGAATTCTATATTGCGGAGTGATCGATG and right GAGGAATTCTGACTTGACCATCAGCGCGGATG. The protein was expressed and the anti-rabbit antibody raised and purified by Proteintech.

\section{Fertility assay}

Single males were mated with two virgin female Oregon-R flies. The flies were allowed to mate at $25^{\circ} \mathrm{C}$ for $48 \mathrm{~h}$ before being transferred to a second batch of fresh vials. After another $48 \mathrm{~h}$, the female flies were removed, and males were dissected to examine the testes for sperm motility. During the period of 10-19 days after the removal of the flies, the progeny in each vial of the second batch was counted.

\section{Climbing assay}

A group of 10 mated 3-4-day old female flies were placed in a cylinder divided vertically into four 5 -cm quadrants. After a $1 \mathrm{~min}$ recovery period, they were banged to the bottom of the cylinder, and then the height climbed in $10 \mathrm{~s}$ was recorded. The quadrant at the top is given a weight of 4 followed by 3, 2 and 1 to the quadrant at the bottom, and the Climbing Index $=\Sigma$ (the numbers of flies in each quadrant $) \times($ each quadrant weight $) /$ the total number of flies. The assay was repeated for five groups of flies.

\section{Larval hearing assay}

Groups of five third instar larvae were placed on a grape juice agar plate positioned on a speaker. After $15 \mathrm{~s}$, a $1 \mathrm{~s}$ tone of $1000 \mathrm{~Hz}$ was delivered by the speaker. For each larva, body contraction was scored (yes $=1 ;$ no $=0$ ) during a $1 \mathrm{~s}$ interval prior to the tone and during the tone. Each larva was tested three times (at $30 \mathrm{~s}$ intervals). The Response Index for test was the sum of the larval scores $(0-5)$ and these were then averaged for the batch. Mean Response Index was determined from three trials (15 larvae total) for each genotype. Significance of differences in mean response index prior and during the tone for each genotype were analysed by two-way ANOVA followed by Sidak's test for multiple comparisons.

\section{Calcium imaging of larval Ch neurons}

Third instar larvae with Ch neuron-specific Gal4 (iav-Gal4) driving GCaMP (iav-Gal4/UAS-GCaMP6f) were filleted in $\mathrm{Ca}^{2+}$-free Ringer solution $(140 \mathrm{mM} \mathrm{NaCl}, 2 \mathrm{mM} \mathrm{KCl}, 6 \mathrm{mM} \mathrm{MgCl} 2,5 \mathrm{mM}$ Hepes-NaOH, $36 \mathrm{mM}$ sucrose, $\mathrm{pH}$ 7.2). After dissection and washing, saline was replaced with Ringer solution ( $130 \mathrm{mM} \mathrm{NaCl}, 5 \mathrm{mM} \mathrm{KCl}, 2 \mathrm{mM} \mathrm{MgCl} 2,2 \mathrm{mM} \mathrm{CaCl}_{2}$, $5 \mathrm{mM}$ Hepes-NaOH, $36 \mathrm{mM}$ sucrose, $\mathrm{pH}$ 7.2). The fillet prep including the ventral nerve cord (VNC) was imaged using a Zeiss AX10 Examiner A1 fluorescence microscope with a $40 \times$ water immersion objective. Imaging was achieved by a Q-imaging WLS LED illumination unit and Photometrics Prime sCMOS camera. Video was recorded at $20 \mathrm{fps}$ for $40 \mathrm{~s}$. Response was recorded as peak $\Delta \mathrm{F} / \mathrm{F}_{0}(\%)$ of mean fluorescence in a region of interest (ROI) bounding the paired axon terminals of the Ch neurons in the VNC when stimulated by a $1024-\mathrm{Hz}$ tuning fork $(52.4 \pm 2.8 \mathrm{~dB}, n=10$ tones). For each fillet prep, three stimuli were presented at $10 \mathrm{~s}$ intervals. Data were analysed in FIJI and Microsoft Excel. $\mathrm{F}_{0}$ for $\Delta \mathrm{F} / \mathrm{F}_{0}(\%)$ was defined as mean 
F of: ( $0 \mathrm{~s}$ to onset of 1 st peak $)+($ end of 1 st peak to onset of 2 nd peak $)+($ end of 2nd peak to onset of 3rd peak). The 'peaks' used in calculation for mean peak of Dnaaf $3^{\Delta C R}$ homozygotes were derived from mean time(s) of recording that correspond to peaks in Dnaaf $3^{\triangle C R}$ heterozygotes, to allow comparison despite lack of peaks present in the homozygote. Mean response to $1024-\mathrm{Hz}$ stimulation was generated by aligning all peaks in recordings (from $0.5 \mathrm{~s}$ before onset of peak to end of peak $+0.5 \mathrm{~s}$ ) where $\mathrm{F}_{0}$ was defined as mean $\mathrm{F}$ recorded $0.5 \mathrm{~s}$ before onset of peak.

\section{Transmission electron microscopy (TEM)}

Whole heads (proboscis removed to facilitate infiltration) or testes of newly eclosed adult males were removed and rinsed in $0.1 \mathrm{M}$ phosphate buffer ( $\mathrm{pH} 7.4$ ). The samples were fixed in freshly made $2.5 \%$ glutaraldehyde, $2 \%$ paraformaldehyde in $0.1 \mathrm{M}$ sodium phosphate buffer $(\mathrm{pH} 7.4)$ solution overnight at $4^{\circ} \mathrm{C}$. Post fixing, the samples were first rinsed four times and then washed three times for $20 \mathrm{~min}$ in $0.1 \mathrm{M}$ phosphate buffer at room temperature. The head or testes samples were post-fixed and imaged by Tracey Davey at the Electron Microscopy Research Services, Newcastle University Medical School, using a Philips CM100 CompuStage (FEI) microscope and an AMT CCD camera.

\section{Protein expression analysis of testes by MS}

Four replicates of 30 pairs of 3-4-day old testes were dissected for the CG17669 CRISPR homozygous mutant line and the Cas9 injection line (control). Sample processing and analysis of the label-free massspectrometry was carried out as described by (zur Lage et al., 2018). Proteins with average LFQ value $<0.05$ in control testes are shown in Table S1 but excluded from further analysis. Ratios of abundance and $P$ values were calculated and plotted. In this analysis, one replicate of mutant testes was found to contain a low level of wildtype testis contaminant. We included this replicate in the analyses shown, as when this replicate is removed from the analysis, very similar dynein chain ratios were observed but the smaller number of replicates led to larger $P$ values. The mass spectrometry proteomics data have been deposited in the ProteomeXchange Consortium via the PRIDE (Perez-Riverol et al., 2019) partner repository with the dataset identifier PXD025409.

\section{Acknowledgements}

We thank Olaf Tomola for preliminary analysis of the proteomics dataset. We are very grateful to Dr Barry Denholm (University of Edinburgh) for use of his Ca-imaging microscope.

\section{Competing interests}

The authors declare no competing or financial interests.

\section{Author contributions}

Conceptualization: A.P.J.; Methodology: P.z.L., Z.X., I.H., A.B.C., A.P.J.; Validation: A.P.J.; Formal analysis: P.z.L., Z.X., I.H., W.C., A.B.C., A.P.J.; Investigation: P.z.L., Z.X., J.L., I.H., W.C.; Data curation: A.B.C., A.v.K.; Writing - original draft: Z.X., A.P.J.; Writing - review \& editing: P.z.L., A.P.J.; Visualization: P.z.L., Z.X., A.P.J.; Supervision: P.z.L., A.v.K., A.P.J.; Project administration: A.v.K., A.P.J.; Funding acquisition: A.v.K., A.P.J.

\section{Funding}

This work was supported by the Biotechnology and Biological Sciences Research Council (BBSRC) (BB/S000801 to A.J.), by the BBSRC EASTBIO doctoral programme (to I.H.), and by the Wellcome Trust (Multiuser Equipment Grant, 208402/Z/17/Z to A.v.K.).

\section{Data availability}

The mass spectrometry proteomics data are available in the ProteomeXchange Consortium via the PRIDE (Perez-Riverol et al., 2019) partner repository with the dataset identifier PXD025409.

\section{References}

Albert, J. T. and Göpfert, M. C. (2015). Hearing in Drosophila. Curr. Opin. Neurobiol. 34, 79-85. doi:10.1016/i.conb.2015.02.001

Avidor-Reiss, T. and Leroux, M. R. (2015). Shared and distinct mechanisms of compartmentalized and cytosolic ciliogenesis. Curr. Biol. 25, R1143-R1150. doi:10.1016/j.cub.2015.11.001
Basiri, M. L., Ha, A., Chadha, A., Clark, N. M., Polyanovsky, A., Cook, B. and Avidor-Reiss, T. (2014). A migrating ciliary gate compartmentalizes the site of axoneme assembly in drosophila spermatids. Curr. Biol. 24, 2622-2631. doi:10. 1016/j.cub.2014.09.047

Bower, R., Vanderwaal, K., O'toole, E., Fox, L., Perrone, C., Mueller, J., Wirschell, M., Kamiya, R., Sale, W. S. and Porter, M. E. (2009). IC138 defines a subdomain at the base of the 11 Dynein that regulates microtubule sliding and flagellar motility. Mol. Biol. Cell 20, 3055-3063. doi:10.1091/mbc.e09-04-0277

Dietzl, G., Chen, D., Schnorrer, F., Su, K., Barinova, Y., Fellner, M., Gasser, B., Kinsey, K., Oppel, S., Scheiblauer, S. et al. (2007). A genome-wide transgenic RNAi library for conditional gene inactivation in Drosophila. Nature 448, 151-156. doi:10.1038/nature05954

Diggle, C. P., Moore, D. J., Mali, G., zur Lage, P., Ait-Lounis, A., Schmidts, M., Shoemark, A., Garcia Munoz, A., Halachev, M. R., Gautier, P. et al. (2014). HEATR2 plays a conserved role in assembly of the ciliary motile apparatus. PLoS Genet. 10, e1004577. doi:10.1371/journal.pgen.1004577

Fabian, L. and Brill, J. A. (2012). Drosophila spermiogenesis. Spermatogenesis 2 197-212. doi:10.4161/spmg. 21798

Fatima, R. (2011). Drosophila dynein intermediate chain gene, Dic61B, is required for spermatogenesis. PLOS ONE 6, e27822. doi:10.1371/journal.pone.0027822

Fingerhut, J. and Yamashita, Y. (2020). mRNA localization mediates maturation of cytoplasmic cilia in Drosophila spermatogenesis. J. Cell Biol. 219, e202003084. doi:10.1083/jcb.202003084

Fok, A. K., Wang, H., Katayama, A., Aihara, M. S. and Allen, R. D. (1994). 22 S axonemal dynein is preassembled and functional prior to being transported to and attached on the axonemes. Cell Motil. Cytoskeleton 29, 215-224. doi:10.1002/cm. 970290304

Fowkes, M. E. and Mitchell, D. R. (1998). The role of preassembled cytoplasmic complexes in assembly of flagellar dynein subunits. Mol. Biol. Cell 9, 2337-2347. doi:10.1091/mbc.9.9.2337

Fushiki, A., Kohsaka, H. and Nose, A. (2013). Role of sensory experience in functional development of Drosophila motor circuits. PLOS ONE 8, e62199. doi:10.1371/journal.pone.0062199

Guo, Z., Chen, W., Huang, J., Wang, L. and Qian, L. (2019). Clinical and genetic analysis of patients with primary ciliary dyskinesia caused by novel DNAAF3 mutations. J. Hum. Genet. 64, 711-719. doi:10.1038/s10038-019-0609-1

Han, Y. G., Kwok, B. H. and Kernan, M. J. (2003). Intraflagellar transport is required in drosophila to differentiate sensory cilia but not sperm. Curr. Biol. 13, 1679-1686. doi:10.1016/j.cub.2003.08.034

Huang, J., Zhou, W., Watson, A. M., Jan, Y.-N. and Hong, Y. (2008). Efficient endsout gene targeting in Drosophila. Genetics 180, 703-707. doi:10.1534/ genetics.108.090563

Kakihara, Y. and Houry, W. A. (2012). The R2TP complex: discovery and functions Biochim. Biophys. Acta 1823, 101-107. doi:10.1016/j.bbamcr.2011.08.016

Karak, S., Jacobs, J. S., Kittelmann, M., Spalthoff, C., Katana, R., Sivan-Loukianova, E., Schon, M. A., Kernan, M. J., Eberl, D. F. and Göpfert, M. C. (2015). Diverse roles of axonemal Dyneins in drosophila auditory neuron function and mechanical amplification in hearing. Sci. Rep. 5, 17085. doi:10.1038/srep17085

Kavlie, R. G., Kernan, M. J. and Eberl, D. F. (2010). Hearing in Drosophila requires TilB, a conserved protein associated with ciliary motility. Genetics 185, 177-188. doi:10.1534/genetics.110.114009

King, S. M. (2016). Axonemal dynein arms. Cold Spring Harb. Perspect. Biol. 8 , a028100. doi:10.1101/cshperspect.a028100

Knowles, M. R., Ostrowski, L. E., Loges, N. T., Hurd, T., Leigh, M. W., Huang, L., Wolf, W. E., Carson, J. L., Hazucha, M. J., Yin, W. et al. (2013). Mutations in SPAG1 cause primary ciliary dyskinesia associated with defective outer and inner dynein arms. Am. J. Hum. Genet. 93, 711-720. doi:10.1016/j.ajhg.2013.07.025

Kondo, S. and Ueda, R. (2013). Highly improved gene targeting by germlinespecific Cas9 expression in Drosophila. Genetics 195, 715-721. doi:10.1534 genetics.113.156737

Laurençon, A., Dubruille, R., Efimenko, E., Grenier, G., Bissett, R., Cortier, E., Rolland, V., Swoboda, P. and Durand, B. (2007). Identification of novel regulatory factor $\mathrm{X}(\mathrm{RFX})$ target genes by comparative genomics in Drosophila species. Genome Biol. 8, R195. doi:10.1186/gb-2007-8-9-r195

Lin, J., Yin, W., Smith, M., Song, K., Leigh, M., Zariwala, M., Knowles, M., Ostrowski, L. and Nicastro, D. (2014). Cryo-electron tomography reveals ciliary defects underlying human RSPH1 primary ciliary dyskinesia. Nat. Commun. $\mathbf{5 3}$ 160

Mali, G., Yeyati, P., Mizuno, S., Keighren, M., zur Lage, P., Garcia-Munoz, A., Shimada, A., Takeda, H., Edlich, F., Takahashi, S. et al. (2018). ZMYND10 functions in a chaperone relay during axonemal dynein assembly. Elife 7, e34389. doi:10.7554/eLife.34389

Mitchison, H. M. and Valente, E. M. (2017). Motile and non-motile cilia in human pathology: from function to phenotypes. J. Pathol. 241, 294-309. doi:10.1002/ path.4843

Mitchison, H. M., Schmidts, M., Loges, N. T., Freshour, J., Dritsoula, A. Hirst, R. A., O'Callaghan, C., Blau, H., Al Dabbagh, M., Olbrich, H. et al. (2012). Mutations in axonemal dynein assembly factor DNAAF3 cause primary ciliary dyskinesia. Nat. Genet. 44, 381-389. doi:10.1038/ng.1106 
Moore, D. J., Onoufriadis, A., Shoemark, A., Simpson, M. A., Zur Lage, P. I., De Castro, S. C., Bartoloni, L., Gallone, G., Petridi, S., Woollard, W. J. et al (2013). Mutations in ZMYND10, a gene essential for proper axonemal assembly of inner and outer dynein arms in humans and flies, cause primary ciliary dyskinesia. Am. J. Hum. Genet. 93, 346-356. doi:10.1016/j.ajhg.2013.07.009

Morris, C., Benson, E. and White-Cooper, H. (2009). Determination of gene expression patterns using in situ hybridization to Drosophila testes. Nat. Protoc. 4 1807-1819. doi:10.1038/nprot.2009.192

Newton, F. G., zur Lage, P. I., Karak, S., Moore, D. J., Göpfert, M. C. and Jarman, A. P. (2012). Forkhead transcription factor Fd3F cooperates with Rfx to regulate a gene expression program for mechanosensory cilia specialization. Dev. Cell 22, 1221-1233. doi:10.1016/j.devcel.2012.05.010

Oda, T., Yagi, T., Yanagisawa, H. and Kikkawa, M. (2013). Identification of the outer-inner dynein linker as a hub controller for axonemal dynein activities. Curr. Biol. 23, 656-664. doi:10.1016/j.cub.2013.03.028

Omran, H., Kobayashi, D., Olbrich, H., Tsukahara, T., Loges, N. T., Hagiwara, H., Zhang, Q., Leblond, G., O'Toole, E., Hara, C. et al. (2008). Ktu/PF13 is required for cytoplasmic pre-assembly of axonemal dyneins. Nature 456, 611-616. doi:10. 1038/nature07471

Paff, T., Loges, N. T., Aprea, I., Wu, K., Bakey, Z., Haarman, E. G., Daniels, J. M. A., Sistermans, E. A., Bogunovic, N., Dougherty, G. W. et al. (2017). Mutations in PIH1D3 cause X-Linked primary ciliary dyskinesia with outer and inner dynein arm defects. Am. J. Hum. Genet. 100, 160-168. doi:10.1016/j.ajhg 2016.11.019

Perez-Riverol, Y., Csordas, A., Bai, J., Bernal-Llinares, M., Hewapathirana, S. Kundu, D. J., Inuganti, A., Griss, J., Mayer, G., Eisenacher, M. et al. (2019). The PRIDE database and related tools and resources in 2019: Improving support for quantification data. Nucleic Acids Res. 47, D442-D450. doi:10.1093/nar/ gky1106

Porter, M. E. (2017). Ciliary and flagellar motility and the nexin-dynein regulatory complex. In Dyneins: The Biology of Dynein Motors: Second Edition (ed. S. King), Elsevier Inc., pp. 299-335.
Sarpal, R., Todi, S. V., Sivan-Loukianova, E., Shirolikar, S, Subramanian, N., Raff, E. C., Erickson, J. W., Ray, K. and Eberl, D. F. (2003). Drosophila KAP interacts with the kinesin II motor subunit KLP64D to assemble chordotonal sensory cilia, but not sperm tails. Curr. Biol. 13, 1687-1696. doi:10.1016/j.cub. 2003.09.025

Sitaram, P., Hainline, S. G. and Lee, L. A. (2014). Cytological analysis of spermatogenesis: live and fixed preparations of Drosophila testes. J. Vis. Exp. 83 e51058. doi:10.3791/51058">10.3791/51058

Steinhauer, J., Statman, B., Fagan, J. K., Borck, J., Surabhi, S., Yarikipati, P. Edelman, D. and Jenny, A. (2019). Combover interacts with the axonemal component Rsp3 and is required for Drosophila sperm individualization. Development 146, dev179275. doi:10.1242/dev.179275

Tarkar, A., Loges, N. T., Slagle, C. E., Francis, R., Dougherty, G. W., Tamayo, J. V., Shook, B., Cantino, M., Schwartz, D., Jahnke, C. et al. (2013). DYX1C1 is required for axonemal dynein assembly and ciliary motility. Nat. Genet. 45, 995-1003. doi:10.1038/ng.2707

Vaughan, C. K. (2014). Hsp90 Picks PIKKs via R2TP and Tel2. Structure 22 799-800. doi:10.1016/j.str.2014.05.012

Vieillard, J., Paschaki, M., Duteyrat, J. L., Augière, C., Cortier, E., Lapart, J. A. Thomas, J. and Durand, B. (2016). Transition zone assembly and its contribution to axoneme formation in Drosophila male germ cells. J. Cell Biol. 214, 875-889. doi:10.1083/jcb.201603086

Wang, J.-W., Beck, E. S. and McCabe, B. D. (2012). A modular toolset for recombination transgenesis and neurogenetic analysis of Drosophila. PLOS ONE 7, e42102. doi:10.1371/journal.pone.0042102

zur Lage, P., Stefanopoulou, P., Styczynska-Soczka, K., Quinn, N., Mali, G., von Kriegsheim, A., Mill, P. and Jarman, A. P. (2018). Ciliary dynein moto preassembly is regulated by Wdr92 in association with HSP90 co-chaperone R2TP. J. Cell Biol. 217, 2583-2598. doi:10.1083/jcb.201709026

zur Lage, P., Newton, F. G. and Jarman, A. P. (2019). Survey of the ciliary motility machinery of Drosophila sperm and ciliated mechanosensory neurons reveals unexpected cell-type specific variations: a model for motile ciliopathies. Front Genet. 10, 24. doi:10.3389/fgene.2019.00024 